\title{
Hexane extract from Uncaria sinensis exhibits anti-apoptotic properties against glutamate-induced neurotoxicity in primary cultured cortical neurons
}

\author{
JI-YEON JANG ${ }^{1}$, HA-NEUI KIM ${ }^{1}$, YU-RI KIM ${ }^{1}$, JIN-WOO HONG ${ }^{2}$, YOUNG-WHAN CHOI ${ }^{3}$, \\ YUNG-HYUN CHOI ${ }^{4}$, HWA-KYOUNG SHIN ${ }^{1}$ and BYUNG-TAE CHOI ${ }^{1}$
}

\author{
${ }^{1}$ Division of Meridian and Structural Medicine, ${ }^{2}$ Division of Clinical Medicine I, School of Korean Medicine, \\ Pusan National University, Yangsan 626-870; ${ }^{3}$ Department of Horticultural Bioscience, College of Natural Resources \\ and Life Science, Pusan National University, Miryang 626-706; ${ }^{4}$ Department of Biochemistry, \\ College of Oriental Medicine, Dongeui University, Busan 614-052, Republic of Korea
}

Received June 25, 2012; Accepted August 29, 2012

DOI: $10.3892 /$ ijmm.2012.1134

\begin{abstract}
We explored the neuroprotective effects of a hexane extract from Uncaria sinensis (HEUS) against glutamateinduced toxicity focusing on its anti-apoptotic mechanism in primary cultured cortical neurons. Pretreatment with HEUS resulted in significantly reduced glutamate-induced toxicity in a dose-dependent manner with a decrease in the release of lactate dehydrogenase. Morphological assay and flow cytometry were performed for determination of the type of cell death; according to the results, treatment with HEUS resulted in a significant reduction of glutamate-induced apoptotic cell death. We examined the anti-apoptotic mechanism of HEUS; treatment with HEUS resulted in markedly decreased expression of death receptor (DR)4, which was induced by glutamate stimulation. In contrast, treatment with HEUS resulted in significantly enhanced levels of expression of glutamate-attenuated XIAP and Bcl-2, as well as marked blockade of glutamate-induced Bid cleavage, which inhibits both extrinsic and intrinsic apoptosis pathways. In addition, pretreatment with HEUS resulted in almost complete blockade of glutamate-induced activation of caspases-8, -9 and -3 , as well as cleavage of poly (ADP-ribose) polymerase. These findings suggest that the neuroprotective effects of HEUS against glutamate-induced toxicity occur via inhibition of DR4 and expression of anti-apoptotic proteins XIAP and $\mathrm{Bcl}-2$ resulting in effective abrogation of the activation of caspase cascades and promotion of cell survival.
\end{abstract}

Correspondence to: Dr Byung-Tae Choi, Division of Meridian and Structural Medicine, School of Korean Medicine, Pusan National University, Yangsan 626-870, Republic of Korea

E-mail: choibt@pusan.ac.kr

Key words: Uncaria sinensis, glutamate, cortical neurons, death receptor 4, Bcl-2, XIAP, caspase

\section{Introduction}

Glutamate, an important excitatory neurotransmitter, plays a key role in learning and memory in the brain, and its concentration is strictly controlled in the central nervous system (1). However, sudden and excess glutamate stimulation induces neuronal damage and even cell death, known as excitotoxicity $(2,3)$. This process is caused by massive influx of $\mathrm{Ca}^{2+}$ via glutamate receptors into cells, leading to initiation of apoptosis through modulation of several apoptosis-related proteins and activation of caspase cascade $(4,5)$.

Another insult of excessive calcium influx attenuates the mitochondrial membrane potential, leading to enhanced $\mathrm{Ca}^{2+}$ permeability through membrane depolarization of mitochondria and release of reactive oxygen species that can confer apoptosis (6). Findings from accumulating studies have suggested an association of glutamate-induced excitotoxicity with several neurodegenerative diseases or brain injuries, including ischemia, Alzheimer's and Huntington's disease (7-9). Therefore, for identification of therapies and prevention of these diseases, further studies of neuroprotection against glutamate-induced excitotoxicity is needed.

Uncaria sinensis (Oliv.) Havil, a member of the Rubiaceae family, is widely used in traditional medicine; in particular, in East Asian countries. Its hooks with stems are known to provide relief from various nervous-related symptoms, including dizziness associated with hypertension, dementia, and epilepsy (10-12). In recent years, studies reporting on plants of the Uncaria genus as pharmacological medicine have been consistent; in particular, several alkaloids and phenolic compounds of Uncaria species have been reported to have antihypertensive and neuroprotective activities both in vivo and in vitro $(13,14)$. The phenolic compound isolated from $U$. sinensis has been reported to exert strong protective effects against glutamate-induced neuronal death (15).

Despite its multiple pharmacological properties, including neuroprotective activities, its precise molecular mechanism has not been well studied. We prepared a hexane extract of U. sinensis (HEUS), which was found to exert protective 
effects against cerebral ischemic damage (16). However, these protective effects of HEUS have not been investigated with regard to the type of neuronal cell death and its related signaling pathway. Therefore, we explored the neuroprotective effects of HEUS against glutamate-induced neurotoxicity and attempted to clarify its relative mechanism for neuronal death in primary cultured rat cortical cells.

\section{Materials and methods}

Preparation of HEUS. The dried hooks and stems of $U$. sinensis were purchased in September 2010 from Hwalim Natural Drugs (Busan, Korea) and were authenticated by Professor J.W. Hong, Division of Clinical Medicine I, School of Korean Medicine, Pusan National University. A voucher specimen (accession number PDRLJG-1) was deposited at the Plant Drug Research Laboratory of Pusan National University (Miryang, Korea). The dried hooks and stems of $U$. sinensis $(1.0 \mathrm{~kg})$ were ground to a fine powder, followed by successive extraction at room temperature with $\mathrm{n}$-hexane, ethyl acetate and methanol. Briefly, filtration and evaporation of hexane extracts of $U$. sinensis were performed under reduced pressure at $45^{\circ} \mathrm{C}$, followed by lyophilization. A white powder of HEUS ( $7.27 \mathrm{~g}$ ) was obtained. Sequential extraction of the remaining powder was performed using ethyl acetate and methanol to yield ethyl acetate extracts of $U$. sinensis (10.68 g) and methanol extracts of $U$. sinensis (31.66 g), respectively. Finally, the solid form of the extract was dissolved with dimethyl sulfoxide (DMSO) for use as HEUS in experiments.

Chemicals and antibodies. Cytosine- $\beta$-D-arabinose furanoside, Hoechst 33342, L-glutamate, 3-(4,5-dimethylthiazol-2-yl)2,5-diphenyltetrazolium bromide (MTT), poly-L-lysine, and $\beta$-actin antibody were purchased from Sigma-Aldrich (St. Louis, MO, USA). Dulbecco's modified Eagle's medium (DMEM), fetal bovine serum (FBS), neurobasal medium, B27, and other neuronal cell culture reagents were purchased from Gibco-Invitrogen (Carlsbad, CA, USA). For western blot analysis, antibodies recognizing DR4, DR5, Bcl-2, Bax, Bid, XIAP, caspases-3, -8, -9, and poly (ADP-ribose) polymerase (PARP) were supplied by Santa Cruz Biotechnology (Santa Cruz, CA, USA), and cIAP-1 and CIAP-2 were supplied by Calbiochem (Cambridge, MA, USA). Secondary antibodies were supplied by Santa Cruz Biotechnology. Caspase colorimetric assay kits for caspases-3, 8 and 9 were purchased from R\&D Systems (Minneapolis, MN, USA) and a FITC Annexin V apoptosis dectection kit was purchased from BD Bioscience (San Diego, CA, USA). A lactate dehydrogenase (LDH) cytotoxicity assay kit and terminal deoxynucleotidyl transferase-mediated dUTP nick end labeling (TUNEL) assay kit were purchased from Promega (Madison, WI, USA).

Primary cortical cell culture. A previously described protocol with some modifications was used for preparation of primary cultures of cortical neurons from fetal rats (17). Briefly, E18-E19 Sprague-Dawley (SD) rat fetuses (DooYeol Biotech, Seoul, Korea) were sacrificed by cervical dislocation under anesthesia. All procedures used in these studies followed the guidelines of protocols approved by the Pusan National University Animal Care and Use Committee in accordance with the National Institutes of Health Guidelines. The cerebral cortex was dissected and chopped into small pieces and digested in $0.25 \%$ trypsin for $15 \mathrm{~min}$ in $\mathrm{Ca}^{2+}$ and $\mathrm{Mg}^{+}$-free Hanks' balanced salt solution (HBSS), followed by mechanical dissociation using a pasteur pipette. After centrifugation, cells were re-suspended in DMEM supplemented with $10 \%$ FBS, $1 \mathrm{mM}$ pyruvate, $4.2 \mathrm{mM}$ sodium bicarbonate, $20 \mathrm{mM}$ HEPES, $0.3 \mathrm{~g} / \mathrm{l}$ bovine serum albumin, and $1 \%$ penicillin/streptomycin at a density of $1 \times 10^{6}$ cells $/ \mathrm{ml}$ and grown on poly-L-lysinecoated plates in a $5 \% \mathrm{CO}_{2}$ humidified incubator at $37^{\circ} \mathrm{C}$. Forty eight hours after plating, cytosine- $\beta$-D-arabinose furanoside was added in order to inhibit proliferation of non-neuronal cells. After three days in culture, the medium was replaced with neurobasal medium containing $2 \%$ B27 supplement, $0.5 \mathrm{mM}$ glutamine, and $1 \%$ penicillin/streptomycin. Cortical neurons were maintained for 9-10 days in primary culture for use in the experiments.

MTT assay. The MTT assay was used for assessment of primary cortical cell viability. Cortical neurons were seeded in 96-well plates at a density of $5 \times 10^{4} /$ well and incubated for $24 \mathrm{~h}$ prior to experimental treatments. After incubation, cells were treated with $200 \mu \mathrm{M}$ of glutamate for $6 \mathrm{~h}$, followed by treatment with $0.5 \mathrm{mg} / \mathrm{ml}$ MTT for $4 \mathrm{~h}$ at $37^{\circ} \mathrm{C}$. Formazan crystals formed in cells were solubilized in DMSO and the absorbance was measured at $570 \mathrm{~nm}$ using a SpectraMax 190 spectrophotometer (Molecular Devices, Sunnyvale, CA, USA). Results were expressed as a percentage of the control.

LDH assay. Cytotoxicity was measured quantitatively according to release of LDH in the culture medium of cell samples. After treatment, cortical neurons were lysed by addition of lysis solution, followed by incubation at $37^{\circ} \mathrm{C}$ for 45-60 min. Sample supernatants were transferred to a 96-well enzymatic assay plate, followed by addition of substrate to each supernatant sample. The enzymatic reaction was allowed to proceed for $30 \mathrm{~min}$ at room temperature, protected from light. After the enzymatic reaction was stopped, the plate was read at $490 \mathrm{~nm}$ using a SpectraMax 190 spectrophotometer. Data represent the percentage of LDH released relative to controls.

Flow cytometric analysis. After treatment, cortical neurons were harvested and cells were washed twice with cold PBS, followed by re-suspension in binding buffer at a concentration of $1 \times 10^{6}$ cells $/ \mathrm{ml} ; 100 \mu 1$ of the solution was transferred to a flow cytometric tube, followed by double staining with Annexin V-FITC and propidium iodide (PI) in the dark at room temperature for $15 \mathrm{~min}$. Subsequently, $400 \mu 1$ of binding buffer was added and analysis of the samples was performed using a flow cytometer (FACSCanto ${ }^{\mathrm{TM}}$ II; Becton-Dickinson, San Jose, CA, USA).

Hoechst staining. Cortical neurons were grown on poly-Llysine-coated glass coverslips at a density of $5 \times 10^{4}$ cells $/ \mathrm{ml}$. After treatment, the cells were fixed in $4 \%$ paraformalehyde for $20 \mathrm{~min}$ at $4^{\circ} \mathrm{C}$. Fixed cells were washed with PBS three times, followed by staining with $10 \mu \mathrm{g} / \mathrm{ml}$ Hoechst 33342 in PBS for 10 min at $37^{\circ} \mathrm{C}$. The cells were washed with PBS three times and mounted in the medium for fluorescence 
(Vector Laboratories, Inc.), followed by observation under a laser scanning confocal microscope (LSM 510; Carl Zeiss, Inc., Oberkochen, Germany). Data are presented as the ratio of chromosomal condensation and morphological change as a percentage of total cells.

TUNEL assay. Cortical neurons were grown on Lab-Tek ${ }^{\circledR}$ chamber slides (Thermo Fisher Scientific, Inc., Rochester, $\mathrm{NY}$, USA) at a density of $1 \times 10^{5}$ cells $/ \mathrm{ml}$. After treatment, the cells were fixed in $4 \%$ methanol-free formaldehyde solution in PBS (pH 7.4) for $25 \mathrm{~min}$ at $4^{\circ} \mathrm{C}$. Fixed cells were then washed with PBS, followed by incubation with DNA-labeling solution at $37^{\circ} \mathrm{C}$ for 60 min inside a humidified chamber to allow the tailing reaction to occur. The DNA-labeling reaction was terminated by $2 \mathrm{X} \mathrm{SSC}$, and followed by washing for removal of unincorporated fluorescein-12-dUTP. The cells were then counterstained with $1 \mu \mathrm{g} / \mathrm{ml}$ PI solution in PBS for $15 \mathrm{~min}$ at room temperature in the dark. Apoptotic neurons were determined by localization of green fluorescent cells (fluorescein-12-dUTP) on a red background using a fluorescence microscope (Carl Zeiss, Inc.). Data are presented as apoptotic cells as a percentage of total cells.

Western blot analysis. After treatment, cortical neurons were washed in cold PBS buffer, followed by homogenization in lysis buffer [200 mM Tris (pH 8.0), $150 \mathrm{mM} \mathrm{NaCl}$, $2 \mathrm{mM}$ EDTA, $1 \mathrm{mM}$ NaF, $1 \%$ NP-40, $1 \mathrm{mM}$ PMSF, $1 \mathrm{mM}$ $\mathrm{Na}_{3} \mathrm{Vo}_{4}$, protease inhibitor cocktail]. Equal amounts of proteins were then separated by $10-12 \%$ sodium dodecyl sulfate-polyacrylamide gel electrophoresis (SDS-PAGE), after which the resolved proteins were transferred to a nitrocellulose membrane (Whatman GmbH, Dassel, Germany). The membrane was blocked with $5 \%$ skim milk, followed by exposure to the appropriate antibodies. All bands were visualized using horseradish peroxidase-conjugated secondary antibodies and an enhanced chemiluminescence system (Pierce Biotechnology, Inc., Rockford, IL, USA). Results of the western blot assay reported here are representative of at least three experiments.

Caspase assay. After treatment, quantification and homogenization of cortical neurons were performed in order to recognize activation of cellular caspase; $150 \mu \mathrm{g}$ protein in cortical neurons was mixed with extraction buffer $[40 \mathrm{mM}$ HEPES (pH 7.4), 20\% glycerol (v/v), 1 mM EDTA, 0.2\% $\mathrm{NP}-40$, and $10 \mathrm{mM}$ DL-DTT] containing $100 \mu \mathrm{M}$ fluorogenic peptide substrate, followed by dilution with extraction buffer, setting a volume of $100 \mu \mathrm{l} /$ sample in a microtiter plate. Caspase-3, -8 and -9 substrates were used with Asp-GluVal-Asp (DEVD)-p-nitroaniline (pNA), Ile-Glu-Thr-Asp (IETD)-pNA and Leu-Glu-His-Asp (LEHD)-pNA, respectively. Caspase activities were determined by measurement of absorbance $(405 \mathrm{~nm})$ of prepared plates after incubation for a period of $3 \mathrm{~h}$.

Data analysis. All data are expressed as means \pm SEM and analyzed using the SigmaStat statistical program version 11.2 (Systat Software, Inc., San Jose, CA, USA). The paired Student's $t$-test was used for analysis of data. A P-value $<0.05$ was considered to indicate a statistically significant result.

\section{Results}

Treatment with HEUS reduces glutamate-induced cytotoxicity. In order to determine whether HEUS is protective against glutamate-induced toxicity, cell viability was measured by MTT assay. Cortical neurons were pretreated with various concentrations of HEUS (0.1-10 $\mu \mathrm{g})$ for $24 \mathrm{~h}$, followed by exposure to glutamate at a concentration of $200 \mu \mathrm{M}$ for a period of $6 \mathrm{~h}$. Stimulation with glutamate alone resulted in reduced cell viability, $34 \%$ of that of the control, however, pretreatment with HEUS resulted in significantly reduced glutamate-induced toxicity in a dose-dependent manner (Fig. 1A). Next, the protective effects of HEUS were also estimated according to changes in the intracellular release of LDH. The level of LDH release showed a significant increase, to $47.6 \%$, after exposure to glutamate, while treatment with HEUS resulted in a marked decrease in the release of $\mathrm{LDH}$, to 38.4 and $24.9 \%$, at a concentration of 1 and $5 \mu \mathrm{g} / \mathrm{ml}$, respectively (Fig. 1B). The protective effects of HEUS were also confirmed by morphological observation using a phase-contrast microscope. Exposure to glutamate resulted in significant cell insult to the cortical neurons, including diminution of cellular processes and decrease in refraction; these morphological features showed marked recovery when cells were pretreated with $5 \mu \mathrm{g} / \mathrm{ml}$ of HEUS (Fig. 1C). These results indicated that pretreatment with HEUS can exert a protective effect against glutamate-induced toxicity in primary cortical neurons.

Treatment with HEUS reduces glutamate-induced apoptotic neuronal death. Findings from recent studies suggest an association of glutamate-triggered cell death with both apoptotic and necrotic processes (18). In order to determine the type of cell death induced by glutamate, we performed several morphological assessments under our experimental conditions. Upon nuclear staining with Hoechst 33342, cortical neurons showed typical morphological signs of apoptosis, including nuclear and cytoplasmic shrinkage, and chromatin condensation, after exposure to glutamate, compared with control cells, which had regular and round-shaped nuclei. However, treatment with HEUS resulted in a significant reduction in these apoptotic features by $\sim 30.8,22.6,13.2$ and $7.0 \%$ from $33.2 \%$ as observed in the glutamate-treated cells at a concentration of 0.1 , $0.5,1$ and $5 \mu \mathrm{g} / \mathrm{ml}$, respectively (Fig. 2A). Next, we performed TUNEL staining, which is designed for detection of DNA strand breaks and quantitation of apoptosis. Consistent with the results of Hoechst staining, after exposure to glutamate, cortical neurons showed a ratio of nuclear DNA fragmentation in total cells to $37.3 \%$. Treatment with HEUS resulted in a marked reduction in TUNEL-positive cells to 18.0 and $7.0 \%$ at a concentration of 1 and $5 \mu \mathrm{g} / \mathrm{ml}$, respectively (Fig. 2B). We further confirmed our results by performance of flow cytometry with Annexin V FITC/PI staining. The percentage of apoptosis in glutamate-treated neurons was up to $38.2 \%$, while the proportion of necrotic cells showed a slight increase to $10.9 \%$ (Fig. 3). These results indicated that after exposure to glutamate, cortical neurons underwent extensive apoptotic-like deaths under our experimental conditions. However, treatment with HEUS resulted in a significant decrease in the number of glutamate-induced apoptotic cells, from 19.7 to $7.9 \%$, at a concentration of 1 and $5 \mu \mathrm{g} / \mathrm{ml}$, respectively (Fig. 3A). These 
A

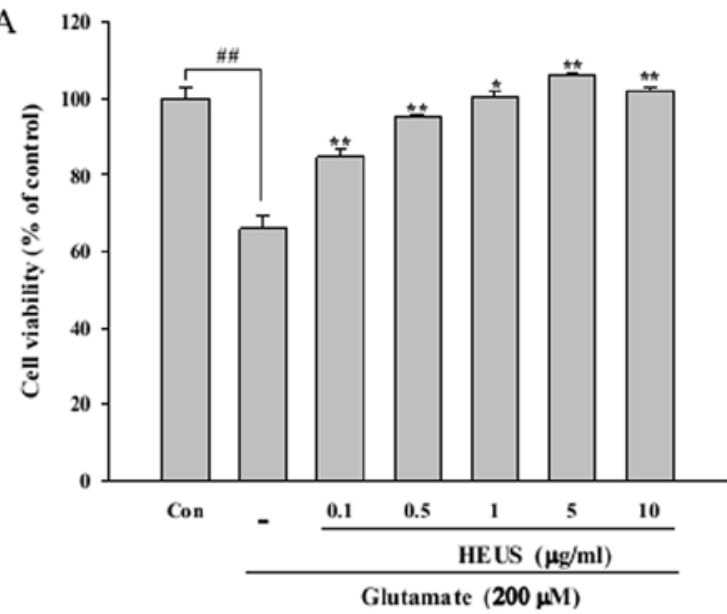

$\mathrm{C}$
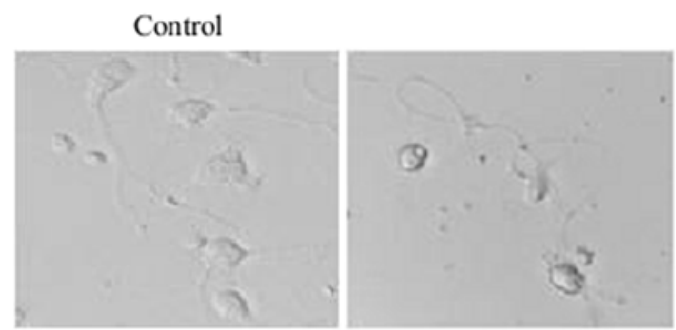

B

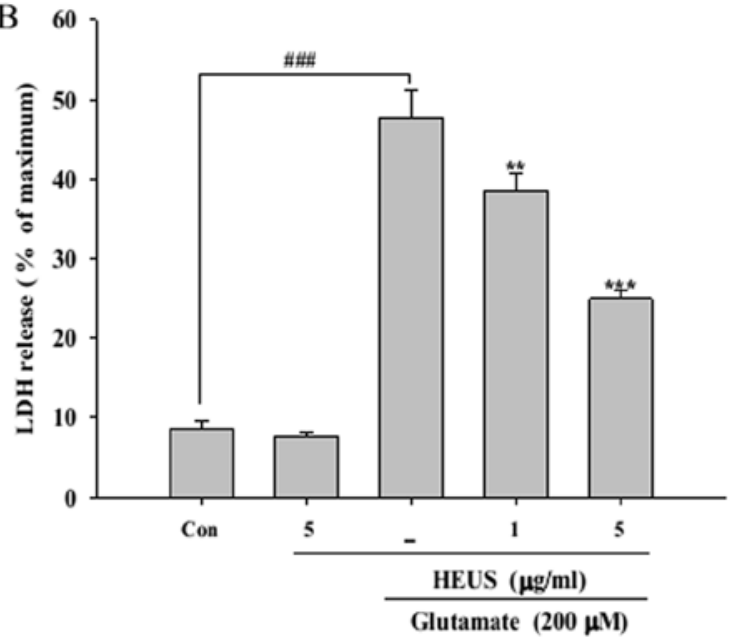

Glutamate $(200 \mu \mathrm{M})$

$\operatorname{HEUS}(1 \mu \mathrm{g} / \mathrm{ml})$

HEUS $(5 \mu \mathrm{g} / \mathrm{ml})$
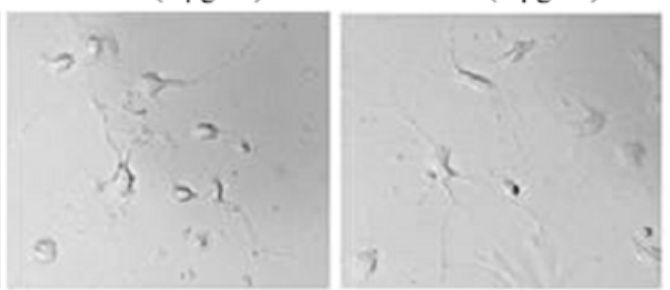

Figure 1. (A) MTT and (B) LDH assays of cell viability and toxicity in glutamate-treated cortical neurons. Cortical neurons were pre-incubated with $0.1,0.5$, 1,5 and $10 \mu \mathrm{g} / \mathrm{ml}$ HEUS for $24 \mathrm{~h}$, followed by exposure to $200 \mu \mathrm{M}$ glutamate for $6 \mathrm{~h} .{ }^{\# \#} \mathrm{P}<0.01,{ }^{\# \# t} \mathrm{P}<0.001$ as compared to the control group; ${ }^{*} \mathrm{P}<0.05$, ${ }^{* *} \mathrm{P}<0.01$ and ${ }^{* * *} \mathrm{P}<0.001$ as compared to the glutamate-treated group. (C) Cell morphology of cortical neurons was assessed by phase contrast microscopy. All data are represented as the means \pm SEM of three independent experiments.
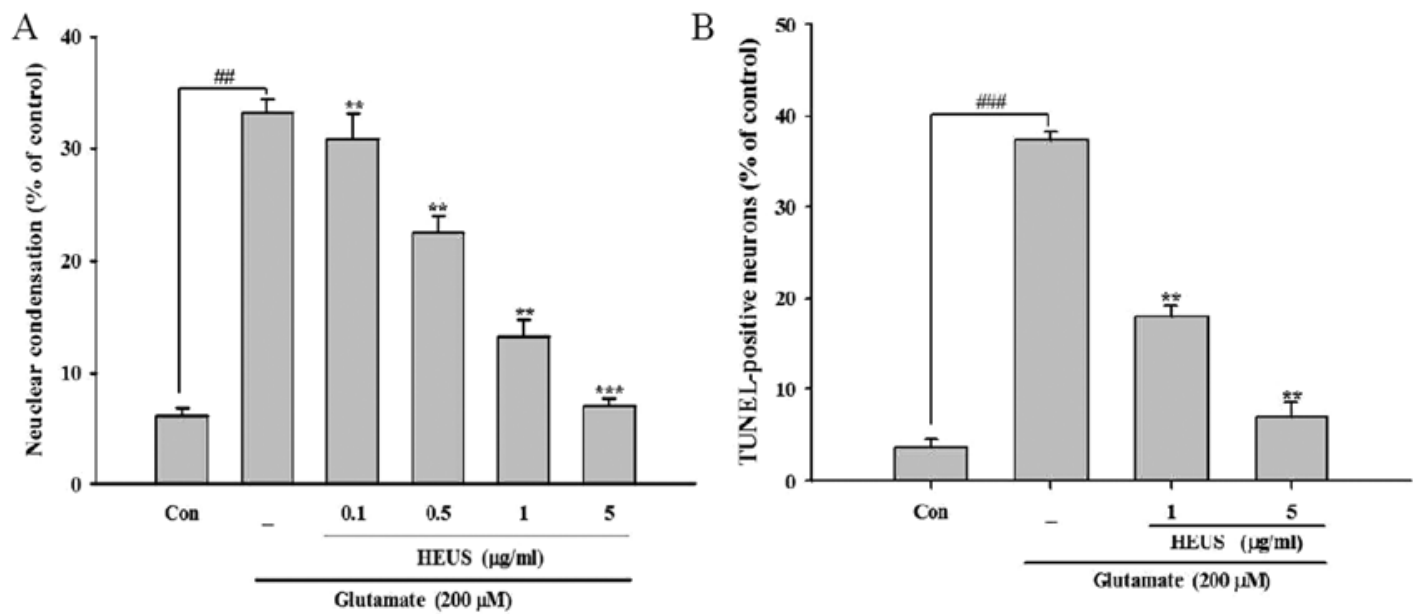

Figure 2. (A) Hoechst 33342 staining and (B) TUNEL assay of apoptotic cells in glutamate-treated cortical neurons. Cortical neurons were pre-incubated with HEUS at the indicated concentrations for $24 \mathrm{~h}$, followed by exposure to $200 \mu \mathrm{M}$ glutamate for $6 \mathrm{~h}$. Quantitative analysis of the histograms expressed as the percentage of apoptotic neurons in the total neurons observed under each microscopic field. Data are represented as the means \pm SEM of three independent experiments. ${ }^{\# \#} \mathrm{P}<0.01,{ }^{\# \# /} \mathrm{P}<0.001$ as compared to the control group; ${ }^{* *} \mathrm{P}<0.01,{ }^{* * *} \mathrm{P}<0.001$ as compared to the glutamate-treated group.

results suggest that pretreatment with HEUS results mainly in protection of cortical cells against glutamate-induced neuronal apoptosis.

HEUS inhibits neuronal apoptosis via expression of DR4 and anti-apoptotic proteins. Mediation of apoptosis occurs primarily through activation of death receptors and/or mito- chondrial signaling (19). Next, using western blot analysis, we evaluated the levels of DRs and apoptosis-related gene expression. Exposure to glutamate resulted in markedly enhanced expression of tumor necrosis factor-related apoptosis-inducing ligand (TRAIL) receptor DR4, whereas, its expression was reduced by pretreatment with HEUS (Fig. 4). Attenuation of TRAIL-induced apoptosis is also known to occur through 
A

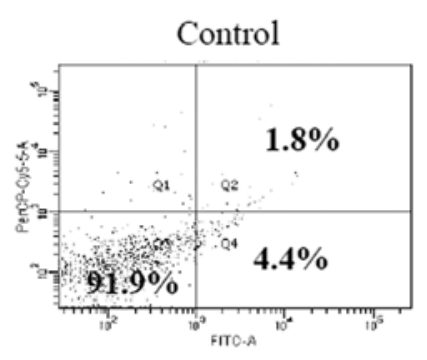

Glutamate $(200 \mu \mathrm{M})$

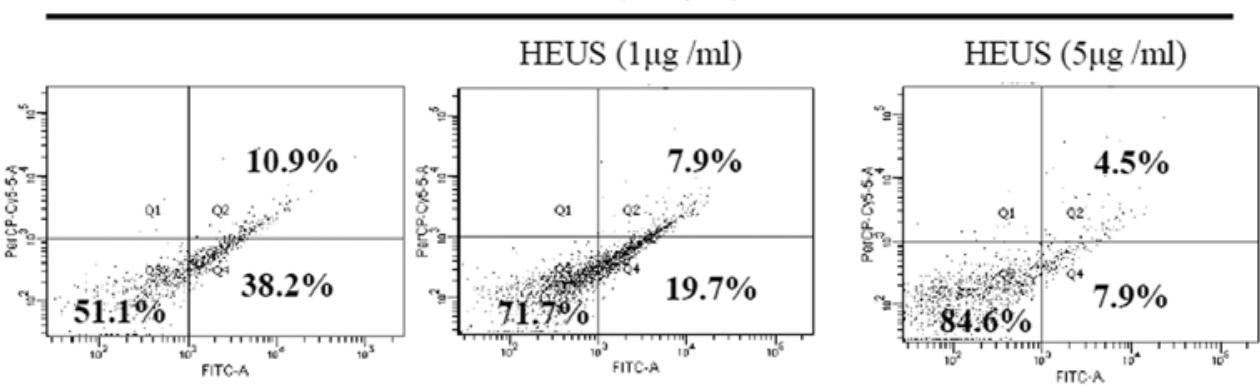

$\mathrm{B}$

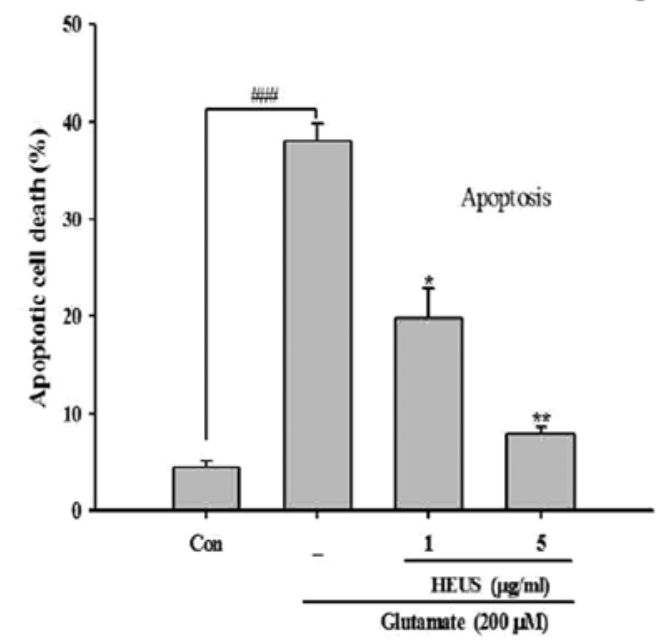

C

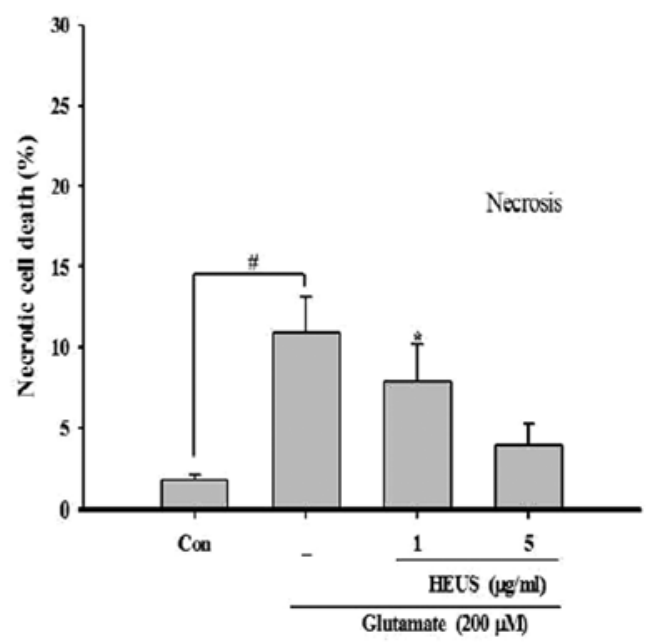

Figure 3. Flow cytometric analysis of the type of cell death in glutamate-treated cortical neurons. Cells were pretreated with HEUS (1 and $5 \mu$ g/ml) for $24 \mathrm{~h}$, followed by exposure to $200 \mu \mathrm{M}$ glutamate for $6 \mathrm{~h}$ and then co-stained with FITC-Annexin V and PI; analysis was performed using a flow cytometer. (A) The four quadrants in the density plots were defined by their fluorescence characteristics [Live cells are Annexin V(-)/PI(-), early apoptotic cells are Annexin V(+)/PI(-) and necrotic or advanced apoptotic cells are both Annexin V(+)/PI(+)]. (B and C) Quantitative analysis of the histograms expressed as the percentage of apoptotic or necrotic cortical neurons in total cortical neurons. ${ }^{\#} \mathrm{P}<0.05,{ }^{\# \# \#} \mathrm{P}<0.001$ as compared to the control group; ${ }^{*} \mathrm{P}<0.05 ;{ }^{* *} \mathrm{P}<0.01$ as compared to the glutamate-treated group. Data are represented as the means \pm SEM of three independent experiments.

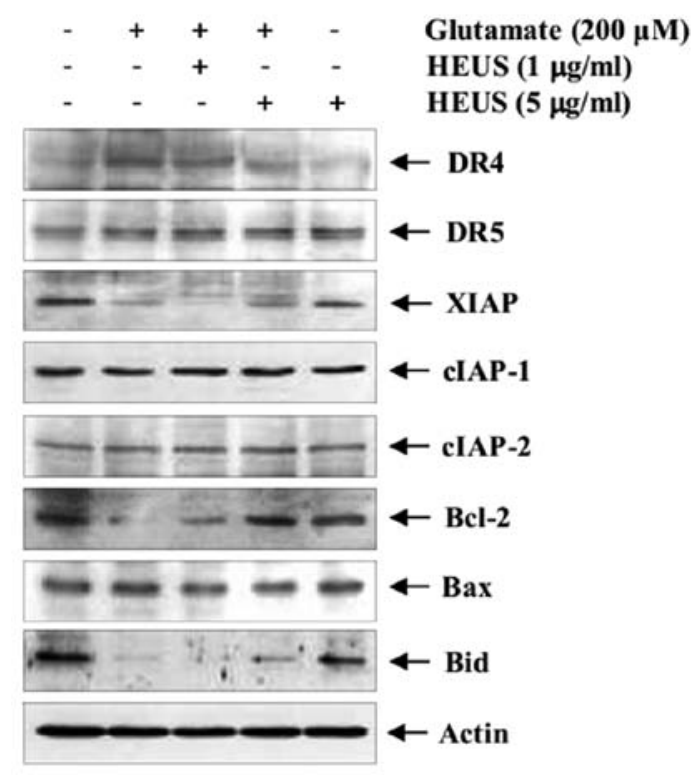

Figure 4. Western blot analysis of apoptosis-related genes in glutamatetreated cortical neurons. Cells were pretreated with HEUS (1 and $5 \mu \mathrm{g} / \mathrm{ml}$ ) for $24 \mathrm{~h}$, followed by exposure to $200 \mu \mathrm{M}$ glutamate for $6 \mathrm{~h}$. Cells were lysed and equal amounts of cellular proteins were subjected to western blot assays using the indicated antibodies. Equal protein loading was confirmed by $\beta$-actin expression. upregulation of numerous anti-apoptotic proteins, including IAPs, which are potent suppressors of apoptosis (19). Thus, we attempted to determine whether the neuroprotective effect of HEUS was involved in expression of IAP family members. Pretreatment with HEUS led to significantly increased expression of glutamate-attenuated XIAP protein, but not cIAPs. Next, we investigated the effect of HEUS on members of the pro- and anti-apoptotic Bcl-2 family, a key regulator of apoptosis. After exposure to glutamate, cortical neurons showed significantly decreased levels of Bcl-2, compared with the control. However, treatment with HEUS resulted in markedly increased glutamate-attenuated anti-apoptotic Bcl-2 expression without alteration of pro-apoptotic Bax expression. Another pro-apoptotic member of the Bcl-2 family, Bid, is cleaved to its truncated active form; tBid induces permeabilization of the outer mitochondrial membrane, leading to promotion of apoptosis (19). Treatment with HEUS resulted in slight recovery of glutamate-attenuated whole Bid expression at a concentration of $5 \mu \mathrm{g} / \mathrm{ml}$. These results suggest that pretreatment with HEUS inhibits neuronal apoptosis via both inhibition of DR4 and activation of anti-apoptotic proteins XIAP and Bcl-2.

Treatment with HEUS reduces activation of glutamateinduced caspases. Eventually, activation of the caspase 
A

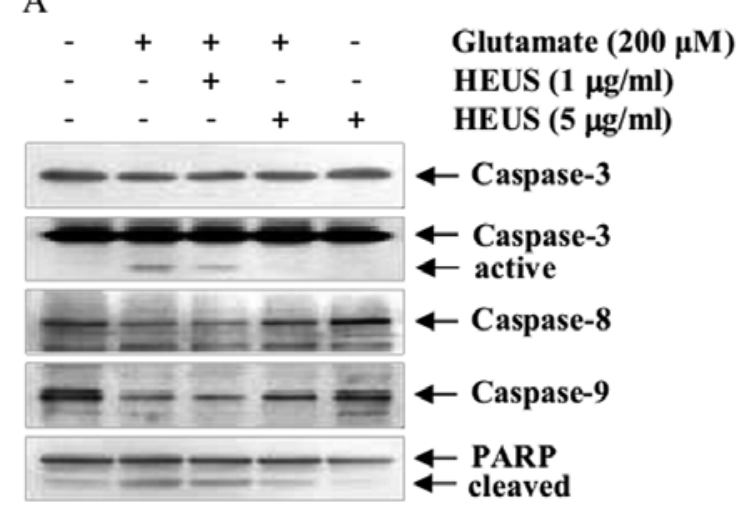

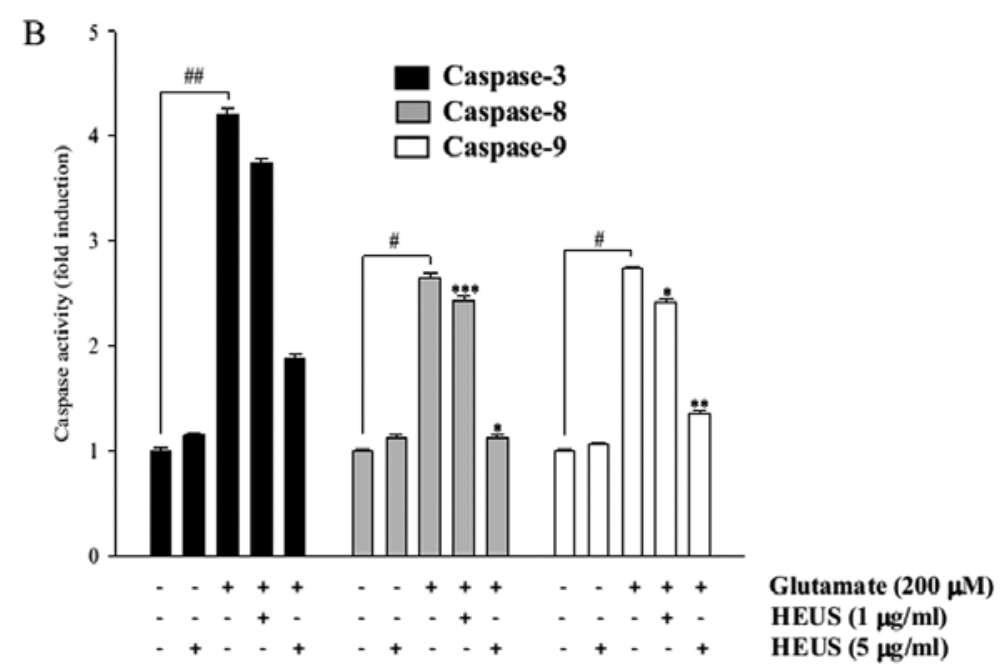

Figure 5. Western blot analysis and caspase activity assay of caspase-3, -8 and -9 and PARP in glutamate-treated cortical neurons. Cells were pretreated with HEUS ( 1 and $5 \mu \mathrm{g} / \mathrm{ml}$ ) for $24 \mathrm{~h}$, followed by exposure to $200 \mu \mathrm{M}$ glutamate for $6 \mathrm{~h}$. (A) Cells were lysed and equal amounts of cellular proteins were subjected to western blotting assays using anti-caspase-3, -8, -9 and PARP antibodies. Equal protein loading was confirmed by $\beta$-actin expression. (B) Quantitative analysis of caspases-3, -8 and -9 activity in vitro. Cell lysates were prepared and assayed for in vitro caspase-3, -8 and -9 activity using DEVD-pNA, IETD-pNA and LEHD-pNA, respectively, as substrates. The concentration of the released fluorescent products was measured. ${ }^{\#} \mathrm{P}<0.05$, ${ }^{\# \#} \mathrm{P}<0.01$ as compared to the control group; ${ }^{*} \mathrm{P}<0.05,{ }^{* *} \mathrm{P}<0.01$ and ${ }^{* * * *} \mathrm{P}<0.001$ as compared to the glutamate-treated group. Data are represented as the means $\pm \mathrm{SEM}$ of three independent experiments.

cascade was induced during the process of glutamate-induced neuronal apoptosis $(5,20)$. Thus we investigated expression and activities of caspase-8, -9 and -3 , which could act either as initiator caspases or as effector caspases, with involvement in both death receptor- and mitochondrial-initiated apoptosis. Western blotting data showed that treatment with HEUS resulted in significantly decreased expression of the cleaved active form of caspase-3 (Fig. 5A). Neither of the active forms of caspase- 8 and -9 was detected in our studies, however, treatment with HEUS induced an increase in their proforms. To further confirm involvement of caspases, we assessed their activities in the lysates of the cells. Exposure to glutamate to cortical neurons resulted in markedly increased activities of caspase-8, -9 and -3 , whereas these activities were significantly attenuated by treatment with HEUS (Fig. 5B). In addition, pretreatment with HEUS resulted in significantly decreased glutamate-enhanced cleavage of PARP, a downstream target of the activated caspase-3 (Fig. 5A). These results suggest that pretreatment with HEUS results in inhibition of neural apoptosis via inhibition of caspase- $8,-9$ and -3 activation.

\section{Discussion}

The controlling mechanisms of glutamate excitotoxicity, a pathological process that causes damage leading to neuronal cell death, have yet to be fully established $(2,3,21)$. In particular, we observed that Uncaria species have been intensively studied for use as a pharmacological medicine against diverse neurological symptoms (13-15). Alkaloids and phenolic compounds from hooks and stems of $U$. sinensis have been reported to exert inhibitory effects against glutamate-induced neuronal death by inhibition of $\mathrm{Ca}^{2+}$ influx in cultured celebellar granule cells (22). The main alkaloid constituent of Uncaria species, rhynchophylline, has been reported to exert strong antihypertensive and neuroprotective activities against cerebral ischemia (13).

However, no studies concerning the precise molecular mechanism of the protective effects of Uncaria species against glutamate-induced neurotoxicity have been reported. We prepared a hexane extract of $U$. sinensis; protection of the brain from ischemic injury by HEUS has been reported in mice, and its mechanism may, in part, be attributed to regulation of Akt/endothelial nitric oxide synthase signaling (16). Findings of our previous studies suggest that HEUS may have a neuroprotective role; therefore, we explored the neuroprotective effect of HEUS against glutamate-induced neurotoxicity in cultured primary cortical cells and investigated the molecular mechanism underlying these effects.

First, we demonstrated that glutamate stimulation resulted in significant induction of toxicity in primary cortical neurons, while pretreatment with HEUS resulted in significantly increased cell viability in a dose-dependent manner (Fig. 1A). These results showed a correlation with those of the LDH release assays (Fig. 1B), and analyses of cell morphology (Fig. 1C) revealed that pretreatment with HEUS effectively protects impaired cortical neurons following glutamate stimulation.

An association of glutamate-induced neuronal death with necrosis and apoptosis, or both, mixed type, depending on the timing and severity of stimulation, has been noted $(18,23)$. Thus, we performed morphological analyses using Hoechst staining, TUNEL assay, and flow cytometric analysis in order to determine whether pretreatment with HEUS prevents either glutamate-induced apoptotic or necrotic cell death. According to our results, glutamate stimulation resulted predominantly in induction of apoptosis, compared to untreated control cells, with only slight elevation of necrosis. In addition, pretreatment with HEUS resulted in effective suppression of glutamateinduced apoptosis (Figs. 2 and 3). These results serve to 
establish that pretreatment with HEUS results in effective inhibition of glutamate-mediated apoptosis in primary cortical neurons, thus exerting a neuroprotective effect.

Some reports have demonstrated the involvement of TRAIL-mediated apoptosis in the pathogenesis of several neurodegenerative diseases of the central nervous system (24,25). Blockade of TRAIL-induced apoptosis in the CNS may be a new molecular therapeutic target for treatment of neurodegenerative diseases. In the present study, we found that glutamate stimulation resulted in elevated levels of TRAIL-mediated receptor DR4, while, pretreatment with HEUS resulted in a critical decrease in its expression (Fig. 4). These results indicated that pretreatment with HEUS inhibits extrinsic/receptor-mediated apoptosis pathways by regulating expression of TRAIL/DR4 signaling. Although these findings do not account for a prominent role in TRAIL-mediated apoptosis, this could be an indication that the anti-apoptotic effect of pretreatment with HEUS might be a good model for investigation of the molecular basis of TRAIL-mediated apoptosis.

Next, we observed that pretreatment with HEUS resulted in significantly enhanced levels of expression of glutamate-attenuated XIAP, Bcl-2, and marked blockade of glutamate-induced cleavage of Bid (Fig. 4). These results indicated that the anti-apoptotic effect of pretreatment with HEUS is not a direct result of altered receptor expression, and that it should induce potent inhibition of both extrinsic and intrinsic apoptosis pathways involved in extrinsic/receptor-mediated cascades (i.e., by downregulation of DR4) and the intrinsic/ mitochondrial-mediated apoptotic pathway (i.e., by upregulation of XIAP, activation of Bcl-2, and downregulation of Bid cleavage). These findings are particularly important since $\mathrm{Bcl}-2$ is known to exert protective action through regulation of mitochondrial membrane potential and to block release of cytochrome $c$ through caspase cleavage (26).

Thus, we attempted to determine whether the anti-apoptotic mechanisms of pretreatment with HEUS were involved in inhibition of caspase activation. Although the exact mechanism of glutamate-induced apoptosis has yet to be fully established, an association of glutamate-induced apoptotic cell death with upregulation of caspase- 3 and its activation has been noted $(20,21)$. In addition, recent studies have reported that use of herbal extracts or their bioactive compounds can result in improvement in the protective activities through modulation of cell survival genes and signaling or downregulation of caspase activity, which is one way of providing protection against neuronal injury $(27,28)$.

Thus, through use of western blot analysis and caspase activity assay, we extend our observation of downstream events of cascase cascade activation. Pretreatment with HEUS resulted in marked attenuation of glutamate-induced caspase-8, -9 and -3 activities, subsequently leading to blocked degradation of PARP-1, caspase-3 target protein (Fig. 5). These results suggest that pretreatment with HEUS can result in strong suppression of glutamate-induced apoptosis through inhibition of a caspase-dependent pathway, which might contribute to the neuroprotective effect of pretreatment with HEUS.

Consequently, we demonstrated that pretreatment with HEUS exerts significant anti-apoptotic effects against glutamate-induced neurotoxicity in primary cultured cortical neurons. Futhermore, these effects are mediated by the regu- lation of both death receptor- and mitochondrial-mediated apoptotic pathways, particularly expression of DR4, XIAP and Bcl-2. Therefore, our findings suggest the potential for HEUS as an attractive candidate for use as a therapeutic approach to treatment of various neurodegenerative diseases associated with glutamate injury.

\section{Acknowledgements}

The present study was supported by the Basic Science Research Program through the National Research Foundation of Korea (NRF) funded by the Ministry of Education, Science and Technology (2011-0022151). This study was financially supported by the 2012 Post-Doctoral Development Program of Pusan National University.

\section{References}

1. Meldrum BS: Glutamate as a neurotransmitter in the brain: review of physiology and pathology. J Nutr 130 (Suppl 4): 1007S-1015S, 2000.

2. Coyle JT and Puttfarcken P: Oxidative stress, glutamate, and neurodegenerative disorders. Science 262: 689-695, 1993.

3. Nishizawa Y: Glutamate release and neuronal damage in ischemia Life Sci 69: 369-381, 2001.

4. Stout AK, Raphael HM, Kanterewicz BI, Klann E and Reynolds IJ: Glutamate-induced neuron death requires mitochondrial calcium uptake. Nat Neurosci 5: 366-373, 1998.

5. Zhang Y and Bhavnani BR: Glutamate-induced apoptosis in primary cortical neurons is inhibited by equine estrogens via down-regulation of caspase- 3 and prevention of mitochondrial cytochrome c release. BMC Neurosci 24: 6-13, 2005.

6. Atlante A, Calissano P, Bobba A, Azzariti A, Marra E and Passarella S: Cytochrome $\mathrm{c}$ is released from mitochondria in a reactive oxygen species (ROS)-dependent fashion and can operate as a ROS scavenger and as a respiratory substrate in cerebellar neurons undergoing excitotoxic death. J Biol Chem 47: 37159-37166, 2000.

7. Paschen W: Glutamate excitotoxicity in transient global cerebral ischemia. Acta Neurobiol Exp (Wars) 56: 313-322, 1996.

8. Hynd MR, Scott HL and Dodd PR: Glutamate-mediated excitotoxicity and neurodegeneration in Alzheimer's disease. Neurochem Int 45: 583-595, 2004.

9. May PC and Gray PN: The mechanism of glutamate-induced degeneration of cultured Huntington's disease and control fibroblasts. J Neurol Sci 70: 101-112, 1985.

10. Lin YW and Hsieh CL: Oral Uncaria rhynchophylla (UR) reduces kainic acid-induced epileptic seizures and neuronal death accompanied by attenuating glial cell proliferation and S100B proteins in rats. J Ethnopharmacol 135: 313-320, 2011.

11. Kuramochi T, Chu J and Suga T: Gou-teng (from Uncaria rhynchophylla Miquel)-induced endothelium-dependent and -independent relaxations in the isolated rat aorta. Life Sci 26: 2061-2069, 1994.

12. Itoh T, Shimada Y and Terasawa K: Efficacy of Choto-san on vascular dementia and the protective effect of the hooks and stems of Uncaria sinensis on glutamate-induced neuronal death. Mech Ageing Dev 111: 155-173, 1999.

13. Zhou J and Zhou S: Antihypertensive and neuroprotective activities of rhynchophylline: the role of rhynchophylline in neurotransmission and ion channel activity. J Ethnopharmacol 132: $15-27,2010$.

14. Suk K, Kim SY, Leem K, Kim YO, Park SY, Hur J, Baek J, Lee KJ, Zheng HZ and Kim H: Neuroprotection by methanol extract of Uncaria rhynchophylla against global cerebral ischemia in rats. Life Sci 70: 2467-2480, 2002

15. Shimada Y, Goto H, Kogure T, Shibahara N, Sakakibara I, Sasaki H and Terasawa K: Protective effect of phenolic compounds isolated from the hooks and stems of Uncaria sinensis on glutamateinduced neuronal death. Am J Chin Med 29: 173-180, 2001.

16. Park SH, Kim JH, Park SJ, Bae SS, Choi YW, Hong JW, Choi BT and Shin HK: Protective effect of hexane extracts of Uncaria sinensis against photothrombotic ischemic injury in mice. $\mathrm{J}$ Ethnopharmacol 138: 774-779, 2011. 
17. Brewer GJ, Torricelli JR, Evege EK and Price PJ: Optimized survival of hippocampal neurons in B27-supplemented Neurobasal, a new serum-free medium combination. J Neurosci Res 35: 567-576, 1993

18. Nicotera P, Ankarcrona M, Bonfoco E, Orrenius S and Lipton SA: Neuronal necrosis and apoptosis: two distinct events induced by exposure to glutamate or oxidative stress. Adv Neurol 72: 95-101, 1997.

19. Fulda S and Debatin KM: Extrinsic versus intrinsic apoptosis pathways in anticancer chemotherapy. Oncogene 25: 4798-4811, 2006.

20. Hirashima Y, Kurimoto M, Nogami K, Endo S, Saitoh M, Ohtani O, Nagata T, Muraguchi A and Takaku A: Correlation of glutamate-induced apoptosis with caspase activities in cultured rat cerebral cortical neurons. Brain Res 849: 109-118, 1999.

21. Lau A and Tymianski M: Glutamate receptors, neurotoxicity and neurodegeneration. Pflugers Arch 460: 525-542, 2010.

22. Shimada Y, Goto H, Itoh T, Sakakibara I, Kubo M, Sasaki H and Terasawa K: Evaluation of the protective effects of alkaloids isolated from the hooks and stems of Uncaria sinensis on glutamate-induced neuronal death in cultured cerebellar granule cells from rats. J Pharm Pharmacol 51: 715-722, 1999.

23. Ankarcrona M, Dypbukt JM, Bonfoco E, Zhivotovsky B, Orrenius S, Lipton SA and Nicotera P: Glutamate-induced neuronal death: a succession of necrosis or apoptosis depending on mitochondrial function. Neuron 15: 961-973, 1995.
24. Huang Y, Erdmann N, Peng H, Zhao Y and Zheng J: The role of TNF related apoptosis-inducing ligand in neurodegenerative diseases. Cell Mol Immunol 2: 113-122, 2005.

25. Uberti D, Ferrari-Toninelli G, Bonini SA, Sarnico I, Benarese M, Pizzi M, Benussi L, Ghidoni R, Binetti G, Spano P, Facchetti F and Memo M: Blockade of the tumor necrosis factor-related apoptosis inducing ligand death receptor DR5 prevents betaamyloid neurotoxicity. Neuropsychopharmacology 4: 872-880, 2006.

26. Yang J, Liu X, Bhalla K, Kim CN, Ibrado AM, Cai J, Peng TI, Jones DP and Wang X: Prevention of apoptosis by Bcl-2: release of cytochrome c from mitochondria blocked. Science 275: 1129-1132, 1997.

27. Li XY, Liang J, Tang YB, Zhou JG and Guan YY: Ginsenoside Rd prevents glutamate-induced apoptosis in rat cortical neurons. Clin Exp Pharmacol Physiol 37: 199-204, 2010.

28. Wang X, Zhu G, Yang S, Wang X, Cheng H, Wang F, Li X and Li Q: Paeonol prevents excitotoxicity in rat pheochromocytoma PC12 cells via downregulation of ERK activation and inhibition of apoptosis. Planta Med 77: 1695-1701, 2011. 